\title{
ON THE CAUCHY PROBLEM FOR GROSS-PITAEVSKII HIERARCHIES
}

\author{
ZEQIAN CHEN AND CHUANGYE LIU
}

\begin{abstract}
The purpose of this paper is to investigate the Cauchy problem for the Gross-Pitaevskii infinite linear hierarchy of equations on $\mathbb{R}^{n}$, $n \geq 1$. We prove local existence and uniqueness of solutions in certain Sobolev type spaces $\mathrm{H}_{\xi}^{\alpha}$ of sequences of marginal density operators with $\alpha>n / 2$. In particular, we give a clear discussion of all cases $\alpha>n / 2$, which covers the local well-posedness problem for Gross-Pitaevskii hierarchy in this situation.
\end{abstract}

\section{INTRODUCTION}

Motivated by recent experimental realizations of Bose-Einstein condensation the theory of dilute, inhomogeneous Bose systems is currently a subject of intensive studies in physics [5]. The ground state of bosonic atoms in a trap has been shown experimentally to display Bose-Einstein condensation (BEC). This fact is proved theoretically by Lieb et al $[15,16,17]$ for bosons with two-body repulsive interaction potentials in the dilute limit, starting from the basic Schrödinger equation. On the other hand, it is well known that the dynamics of Bose-Einstein condensates are well described by the Gross-Pitaevskii equation $[12,13,18]$. A rigorous derivation of this equation from the basic many-body Schrödinger equation in an appropriate limit is not a simple matter, however, and has only achieved recently in three spatial dimensions by Elgart, Erdös, Schlein and Yau [6, 7, 8, 9, 10, 11], based on the notion of so-called Gross-Pitaevskii hierarchies. In their program an important step is to prove uniqueness to the Gross-Pitaevskii hierarchy via Feynman graph (see $[8,14]$ ).

Recently, T.Chen and N.Pavlović [2] started to investigate the Cauchy problem for the Gross-Pitaevskii hierarchy, using a Picard-type fixed point argument. In the present paper, we continue this line of investigation. We will prove local existence and uniqueness of solutions in certain Sobolev type spaces $\mathrm{H}_{\xi}^{\alpha}$ (for definition see Section 2 below) of sequences of marginal density operators with $\alpha>n / 2$. Instead of using a fixed point principle as in [2], here we use the fully expanded iterated Duhamel series, and a

2010 Mathematics Subject Classification: 35Q55, 81V70.

Key words: Gross-Pitaevskii hierarchy, nonlinear Schrodinger equation, Cauchy problem, Space-time type estimate.

C.Liu is partially supported by NSFC grants No.11071095. 
Cauchy convergence criterion, without additional conditions on any spacetime norms. The assumption of $\alpha>n / 2$ allows us to significantly simplify the approaches and provide an improvement for the previous work [2] in all cases $\alpha>n / 2$. Our proof involves the simple property that the interaction operators $B^{(k)}$ are bounded maps from the $k+1$-particle Hilbert space $\mathrm{H}_{k+1}^{\alpha}$ to the $k$-particle Hilbert space $\mathrm{H}_{k}^{\alpha}$ in the cubic case. A case of this type has previously been presented by Chen-Pavlović [4] in their derivation of the quintic NLS for $n=1$. In the much more difficult situation $\alpha \leq n / 2$, as done recently in [3], it is necessary to invoke the Strichartz estimates of the type introduced in the pioneering work of Klainerman-Machedon [14].

The paper is organized as follows. In Section 2, some notations and the main result are presented. Section 3 is devoted to present elementary estimates which will be used later. In particular, we will prove the fact that the interaction operators $B^{(k)}$ are bounded maps from the $k+1$-particle Hilbert space $\mathrm{H}_{k+1}^{\alpha}$ to the $k$-particle Hilbert space $\mathrm{H}_{k}^{\alpha}$ in the cubic case for $\alpha>n / 2$. The proof is completely analogous to that of the classical Sobolev inequality $\|f\|_{L^{\infty}\left(\mathbb{R}^{n}\right)} \leq C\|f\|_{\mathrm{H}^{\alpha}\left(\mathbb{R}^{n}\right)}$. In section 4 , the main result is proved. Finally, in Section 5, we discuss the so-called quintic GrossPitaevskii hierarchy and extend the result obtained in the previous sections to that case.

\section{Preliminaries and statement of the main Result}

2.1. Gross-Pitaevskii hierarchies. As follows, we denote by $x$ a general variable in $\mathbb{R}^{n}$ and by $\mathbf{x}=\left(x_{1}, \cdots, x_{N}\right)$ a point in $\mathbb{R}^{N n}$. We will also use the notation $\mathbf{x}_{k}=\left(x_{1}, \ldots, x_{k}\right) \in \mathbb{R}^{k n}$ and $\mathbf{x}_{N-k}=\left(x_{k+1}, \ldots, x_{N}\right) \in \mathbb{R}^{(N-k) n}$. For a function $f$ on $\mathbb{R}^{k n}$ we let

$$
\left(\Theta_{\sigma} f\right)\left(x_{1}, \ldots, x_{k}\right)=f\left(x_{\sigma(1)}, \ldots, x_{\sigma(k)}\right)
$$

for any permutation $\sigma \in \Pi_{k}\left(\Pi_{k}\right.$ denotes the set of permutations on $k$ elements). Then, each $\Theta_{\sigma}$ is a unitary operator on $L^{2}\left(\mathbb{R}^{k n}\right)$. A bounded operator $A$ on $L^{2}\left(\mathbb{R}^{k n}\right)$ is called $k$-partite symmetric or simply symmetric if

$$
\Theta_{\sigma} A \Theta_{\sigma^{-1}}=A
$$

for every $\sigma \in \Pi_{k}$. Evidently, a density operator $\gamma^{(k)}$ on $L^{2}\left(\mathbb{R}^{k n}\right)$ (i.e., $\gamma^{(k)} \geq 0$ and $\left.\operatorname{tr} \gamma^{(k)}=1\right)$ with the kernel function $\gamma^{(k)}\left(\mathbf{x}_{k} ; \mathbf{x}_{k}^{\prime}\right)$ is $k$-partite symmetric if and only if

$$
\gamma^{(k)}\left(x_{1}, \ldots, x_{k} ; x_{1}^{\prime}, \ldots, x_{k}^{\prime}\right)=\gamma^{(k)}\left(x_{\sigma(1)}, \ldots, x_{\sigma(k)} ; x_{\sigma(1)}^{\prime}, \ldots, x_{\sigma(k)}^{\prime}\right)
$$

for any $\sigma \in \Pi_{k}$.

Also, we set

$$
L_{s}^{2}\left(\mathbb{R}^{k n}\right)=\left\{f \in L^{2}\left(\mathbb{R}^{k n}\right): \Theta_{\sigma} f=f, \forall \sigma \in \Pi_{k}\right\},
$$

equipped with the inner product of $L^{2}\left(\mathbb{R}^{k n}\right)$. Clearly, $L_{s}^{2}\left(\mathbb{R}^{k n}\right)$ is a Hilbert subspace of $L^{2}\left(\mathbb{R}^{k n}\right)$. It is easy to check that any $k$-partite symmetric operator on $L^{2}\left(\mathbb{R}^{k n}\right)$ preserves $L_{s}^{2}\left(\mathbb{R}^{k n}\right)$. 
Definition 2.1. Given $n \geq 1$, the $n$-dimensional Gross-Pitaevskii (GP) hierarchy refers to a sequence $\left\{\gamma_{t}^{(k)}\right\}_{k \geq 1}$ of $k$-partite symmetric density operators on $L^{2}\left(\mathbb{R}^{k n}\right)$, where $t \geq 0$, which satisfy the Gross-Pitaevskii infinite linear hierarchy of equations,

$$
i \partial_{t} \gamma_{t}^{(k)}=\left[-\Delta^{(k)}, \gamma_{t}^{(k)}\right]+\mu B^{(k)} \gamma_{t}^{(k+1)}, \Delta^{(k)}=\sum_{j=1}^{k} \Delta_{x_{j}}, \mu= \pm 1,
$$

with initial conditions

$$
\gamma_{t=0}^{(k)}=\gamma_{0}^{(k)}, \quad k=1,2, \ldots
$$

Here, $\Delta_{x_{j}}$ refers to the usual Laplace operator with respect to the variables $x_{j} \in \mathbb{R}^{n}$ and the operator $B^{(k)}$ is defined by

$$
B^{(k)} \gamma_{t}^{(k+1)}=\sum_{j=1}^{k} \operatorname{tr}_{k+1}\left[\delta\left(x_{j}-x_{k+1}\right), \gamma_{t}^{(k+1)}\right]
$$

where the notation $\operatorname{tr}_{k+1}$ indicates that the trace is taken over the $(k+1)$-th variable.

As in [2], we refer to (2.2) as the cubic GP hierarchy. For $\mu=1$ or $\mu=-1$ we refer to the corresponding GP hierarchies as being defocusing or focusing, respectively. We note that the cubic Gross-Pitaevskii hierarchy accounts for two-body interactions between the Bose particles (e.g., see [5, 10] and references therein for details).

Remark 2.1. In terms of the kernel functions $\gamma_{t}^{(k)}\left(\mathbf{x}_{k} ; \mathbf{x}_{k}^{\prime}\right)$, we can rewrite (2.2) as follows:

$$
\left(i \partial_{t}+\triangle_{ \pm}^{(k)}\right) \gamma_{t}^{(k)}\left(\mathbf{x}_{k} ; \mathbf{x}_{k}^{\prime}\right)=\mu\left[B^{(k)} \gamma_{t}^{(k+1)}\right]\left(\mathbf{x}_{k} ; \mathbf{x}_{k}^{\prime}\right)
$$

where $\triangle_{ \pm}^{(k)}=\sum_{j=1}^{k}\left(\Delta_{x_{j}}-\Delta_{x_{j}^{\prime}}\right)$, with initial conditions

$$
\gamma_{t=0}^{(k)}\left(\mathbf{x}_{k} ; \mathbf{x}_{k}^{\prime}\right)=\gamma_{0}^{(k)}\left(\mathbf{x}_{k} ; \mathbf{x}_{k}^{\prime}\right), \quad k=1,2, \ldots
$$

In particular, the action of $B^{(k)}$ on density operators with smooth kernel functions, $\gamma^{(k+1)}\left(\mathbf{x}_{k+1} ; \mathbf{x}_{k+1}^{\prime}\right) \in \mathcal{S}\left(\mathbb{R}^{(k+1) n} \times \mathbb{R}^{(k+1) n}\right)$, is given by

$$
\begin{aligned}
{\left[B^{(k)} \gamma_{t}^{(k+1)}\right]\left(\mathbf{x}_{k} ; \mathbf{x}_{k}^{\prime}\right)=} & \sum_{j=1}^{k} \int d x_{k+1} d x_{k+1}^{\prime} \gamma_{t}^{(k+1)}\left(\mathbf{x}_{k}, x_{k+1} ; \mathbf{x}_{k}^{\prime}, x_{k+1}^{\prime}\right) \\
& \times \delta\left(x_{k+1}^{\prime}-x_{k+1}\right)\left[\delta\left(x_{j}-x_{k+1}\right)-\delta\left(x_{j}^{\prime}-x_{k+1}\right)\right] .
\end{aligned}
$$

The action of $B^{(k)}$ can be extended to generic density operators. This will be made precise in Lemma 3.2. 
Remark 2.2. Let $\varphi \in \mathrm{H}^{1}\left(\mathbb{R}^{n}\right)$, then one can easily verify that a particular solution to (2.3) with initial conditions

$$
\gamma_{t=0}^{(k)}\left(\mathbf{x}_{k} ; \mathbf{x}_{k}^{\prime}\right)=\prod_{j=1}^{k} \varphi\left(x_{j}\right) \overline{\varphi\left(x_{j}^{\prime}\right)}, \quad k=1,2, \ldots,
$$

is given by

$$
\gamma_{t}^{(k)}\left(\mathbf{x}_{k} ; \mathbf{x}_{k}^{\prime}\right)=\prod_{j=1}^{k} \varphi_{t}\left(x_{j} \overline{\varphi_{t}\left(x_{j}^{\prime}\right)} k=1,2, \ldots,\right.
$$

where $\varphi_{t}$ satisfies the cubic non-linear Schrödinger equation

$$
i \partial_{t} \varphi_{t}=-\Delta \varphi_{t}+\mu\left|\varphi_{t}\right|^{2} \varphi_{t}, \quad \varphi_{t=0}=\varphi
$$

which is defocusing if $\mu=1$, and focusing if $\mu=-1$.

The Gross-Pitaevskii hierarchy (2.2) can be written in the integral form

$$
\gamma_{t}^{(k)}=\mathcal{U}_{0}^{(k)}(t) \gamma_{0}^{(k)}+\int_{0}^{t} d s \mathcal{U}_{0}^{(k)}(t-s) \tilde{B}^{(k)} \gamma_{s}^{(k+1)}, k=1,2, \ldots,
$$

where $\tilde{B}^{(k)}=-i \mu B^{(k)}$. Hereafter, the free evolution operator is defined by

$$
\mathcal{U}_{0}^{(k)}(t) A=\exp \left(i t \Delta^{(k)}\right) A \exp \left(-i t \Delta^{(k)}\right), k=1,2, \ldots,
$$

for every operator $A$ on $L^{2}\left(\mathbb{R}^{k n}\right)$. The action of $\mathcal{U}_{0}^{(k)}(t)$ on kernel functions $\gamma^{(k)} \in L^{2}\left(\mathbb{R}^{k n} \times \mathbb{R}^{k n}\right)$ is given by

$$
\mathcal{U}_{0}^{(k)}(t) \gamma^{(k)}\left(\mathbf{x}_{k}, \mathbf{x}_{k}^{\prime}\right)=e^{-i t \triangle_{ \pm}^{(k)}} \gamma^{(k)}\left(\mathbf{x}_{k}, \mathbf{x}_{k}^{\prime}\right)
$$

Formally we can expand the solution $\gamma_{t}^{(k)}$ of (2.6) for any $m \geq 1$ as

$$
\begin{aligned}
\gamma_{t}^{(k)} & =\mathcal{U}_{0}^{(k)}(t) \gamma_{0}^{(k)}+\sum_{j=1}^{m-1} \int_{0}^{t} d s_{1} \int_{0}^{s_{1}} d s_{2} \cdots \int_{0}^{s_{j-1}} d s_{j} \mathcal{U}_{0}^{(k)}\left(t-s_{1}\right) \tilde{B}^{(k)} \ldots \\
& \times \mathcal{U}_{0}^{(k+j-1)}\left(s_{j-1}-s_{j}\right) \tilde{B}^{(k+j-1)} \mathcal{U}_{0}^{(k+j)}\left(s_{j}\right) \gamma_{0}^{(k+j)} \\
& +\int_{0}^{t} d s_{1} \int_{0}^{s_{1}} d s_{2} \cdots \int_{0}^{s_{m-1}} d s_{m} \mathcal{U}_{0}^{(k)}\left(t-s_{1}\right) \tilde{B}^{(k)} \ldots \\
& \times \mathcal{U}_{0}^{(k+m-1)}\left(s_{m-1}-s_{m}\right) \tilde{B}^{(k+m-1)} \gamma_{s_{m}}^{(k+m)}
\end{aligned}
$$

with the convention $s_{0}=t$. The terms in the summation contain only the initial data. The last error term involves the density operator at an intermediate time $s_{m}$. 
2.2. Statement of the main result. In order to state our main results, we require some more notation. We will use $\gamma^{(k)}, \rho^{(k)}$ for denoting either (density) operators or kernel functions. For $k \geq 1$ and $\alpha>0$, we denote by $\mathrm{H}_{k}^{\alpha}=\mathrm{H}^{\alpha}\left(\mathbb{R}^{k n} \times \mathbb{R}^{k n}\right)$ the space of measurable functions $\gamma^{(k)}=\gamma^{(k)}\left(\mathbf{x}_{k}, \mathbf{x}_{k}^{\prime}\right)$ in $L^{2}\left(\mathbb{R}^{k n} \times \mathbb{R}^{k n}\right)$ such that

$$
\left\|\gamma^{(k)}\right\|_{\mathrm{H}_{k}^{\alpha}}:=\left\|S^{(k, \alpha)} \gamma^{(k)}\right\|_{L^{2}\left(\mathbb{R}^{k n} \times \mathbb{R}^{k n}\right)}<\infty,
$$

where

$$
S^{(k, \alpha)}:=\prod_{j=1}^{k}\left[\left(1-\Delta_{x_{j}}\right)^{\frac{\alpha}{2}}\left(1-\Delta_{x_{j}^{\prime}}\right)^{\frac{\alpha}{2}}\right] .
$$

Evidently, $\mathrm{H}_{k}^{\alpha}$ is a Hilbert space with the inner product

$$
\left\langle\gamma^{(k)}, \rho^{(k)}\right\rangle=\left\langle S^{(k, \alpha)} \gamma^{(k)}, S^{(k, \alpha)} \rho^{(k)}\right\rangle_{L^{2}\left(\mathbb{R}^{k n} \times \mathbb{R}^{k n}\right)} .
$$

Moreover, the norm $\|\cdot\|_{\mathrm{H}_{k}^{\alpha}}$ is invariance under the action of $\mathcal{U}_{0}^{(k)}(t)$, that is,

$$
\left\|\mathcal{U}_{0}^{(k)}(t) \gamma^{(k)}\right\|_{\mathrm{H}_{k}^{\alpha}}=\left\|\gamma^{(k)}\right\|_{\mathrm{H}_{k}^{\alpha}}
$$

because $\exp \left\{ \pm i t \Delta^{(k)}\right\}$ commutate with $\Delta_{x_{j}}$ for any $j$.

Let $0<\xi<1$ and $\alpha>0$, we define

$$
\mathcal{H}_{\xi}^{\alpha}=\left\{\Gamma=\left\{\gamma^{(k)}\right\}_{k \geq 1} \in \bigotimes_{k=1}^{\infty} \mathrm{H}_{k}^{\alpha}:\|\Gamma\|_{\mathcal{H}_{\xi}^{\alpha}}:=\sum_{k=1}^{\infty} \xi^{k}\left\|\gamma^{(k)}\right\|_{\mathrm{H}_{k}^{\alpha}}<\infty\right\} .
$$

Evidently, $\mathcal{H}_{\xi}^{\alpha}$ is a Banach space equipped with the norm $\|\cdot\|_{\mathcal{H}_{\xi}^{\alpha} \text {, which is }}$ introduced in [2]. We remark that similar spaces are used in the isospectral renormalization group analysis of spectral problems in quantum field theory (see [1]).

Definition 2.2. For $T>0, \Gamma_{t}=\left\{\gamma_{t}^{(k)}\right\}_{k \geq 1} \in C\left([0, T], \mathcal{H}_{\xi}^{\alpha}\right)$ is said to be a local (mild) solution to the Gross-Pitaevskii hierarchy (2.2) if for every $k=1,2, \ldots$,

$$
\gamma_{t}^{(k)}=\mathcal{U}_{0}^{(k)}(t) \gamma_{0}^{(k)}+\int_{0}^{t} d s \mathcal{U}_{0}^{(k)}(t-s) \tilde{B}^{(k)} \gamma_{s}^{(k+1)}, \quad \forall t \in[0, T],
$$

holds in $\mathrm{H}_{k}^{\alpha}$.

Our main result in this paper is the following theorem.

Theorem 2.1. Assume that $n \geq 1$ and $\alpha>n / 2$. Suppose $\Gamma_{0}=\left\{\gamma_{0}^{(k)}\right\}_{k \geq 1} \in$ $\mathcal{H}_{\xi}^{\alpha}$ for some $0<\xi<1$. Then there exists a constant $C=C_{\alpha, n}$ depending only on $n$ and $\alpha$ such that, for a fixed $0<T<\xi / C$ with $\eta=\xi-C T$, the following hold.

(i) There exists a solution $\Gamma_{t}=\left\{\gamma_{t}^{(k)}\right\}_{k \geq 1} \in C\left([0, T], \mathcal{H}_{\eta}^{\alpha}\right)$ to the GrossPitaevskii hierarchy (2.2) with the initial data $\Gamma_{0}$ satisfying

$$
\left\|\Gamma_{t}\right\|_{C\left([0, T], \mathcal{H}_{\eta}^{\alpha}\right)} \leq \frac{\eta}{\xi}\left\|\Gamma_{0}\right\|_{\mathcal{H}_{\xi}^{\alpha}}
$$


(ii) For $T=\xi /(5 C)$, if $\Gamma_{t}$ and $\Gamma_{t}^{\prime}$ in $C\left([0, T], \mathcal{H}_{\eta}^{\alpha}\right)$ are two solutions to $(2.2)$ with initial conditions $\Gamma_{t=0}=\Gamma_{0}$ and $\Gamma_{t=0}^{\prime}=\Gamma_{0}^{\prime}$ in $\mathcal{H}_{\xi}^{\alpha}$ respectively, then

$$
\left\|\Gamma_{t}-\Gamma_{t}^{\prime}\right\|_{C\left([0, T], \mathcal{H}_{\eta}^{\alpha}\right)} \leq \frac{4}{5}\left\|\Gamma_{0}-\Gamma_{0}^{\prime}\right\|_{\mathcal{H}_{\xi}^{\alpha}}
$$

Consequently, the solution $\Gamma_{t}$ to the initial problem (2.2) with the initial data in $\mathcal{H}_{\xi}^{\alpha}$ is unique in $C\left([0, T], \mathcal{H}_{\eta}^{\alpha}\right)$ for any $0<T<\xi / C$.

Remark 2.3. We will prove this theorem by the method of infinitely iterating the Duhamel series, and proving Cauchy convergence without additional conditions on spacestime bounds, and however, our argument won't work if $\alpha \leq n / 2$. The assumption of $\alpha>n / 2$ allows us to significantly simplify the approaches in the previous work [2] and improve the corresponding one. In the much more difficult situation $\alpha \leq \frac{n}{2}$, as done recently in [3], it is necessary to invoke the Strichartz estimates of the type introduced in the pioneering work of Klainerman-Machedon [14].

\section{Preliminary estimates}

In the sequel, we will mostly work in Fourier (momentum) space. Following [8], we use the convention that variables $p, q, r, p^{\prime}, q^{\prime}, r^{\prime}$ always refer to $n$ dimensional Fourier variables, while $x, x^{\prime}, y, y^{\prime}, z, z^{\prime}$ denote the position space variables. With this convention, the usual hat indicating the Fourier transform will be omitted. For example, for $k \geq 1$ the kernel of a bounded operator $A$ on $L^{2}\left(\mathbb{R}^{k n}\right)$ in position space is $K\left(\mathbf{x}_{k} ; \mathbf{x}_{k}^{\prime}\right)$, then in the momentum space it is given by the Fourier transform

$$
K\left(\mathbf{q}_{k} ; \mathbf{q}_{k}^{\prime}\right)=\left\langle K, e^{-i\left\langle\cdot, \mathbf{q}_{k}\right\rangle} e^{i\left\langle\cdot, \mathbf{q}_{k}^{\prime}\right\rangle}\right\rangle=\int d \mathbf{x}_{k} d \mathbf{x}_{k}^{\prime} K\left(\mathbf{x}_{k} ; \mathbf{x}_{k}^{\prime}\right) e^{-i\left\langle\mathbf{x}_{k}, \mathbf{q}_{k}\right\rangle} e^{i\left\langle\mathbf{x}_{k}^{\prime}, \mathbf{q}_{k}^{\prime}\right\rangle},
$$

with the slight abuse of notation of omitting the hat on left hand side. Here,

$$
\left\langle\mathbf{x}_{k}, \mathbf{q}_{k}\right\rangle=\sum_{j=1}^{k} x_{j} \cdot q_{j}, \quad \forall \mathbf{x}_{k}=\left(x_{1}, \ldots, x_{k}\right), \mathbf{q}_{k}=\left(q_{1}, \ldots, q_{k}\right) \in \mathbb{R}^{k n}
$$


Thus, on kernels in the momentum space $B^{(k)}$ in (2.4) acts according to

$$
\begin{aligned}
& {\left[B^{(k)} \gamma^{(k+1)}\right]\left(\mathbf{p}_{k} ; \mathbf{p}_{k}^{\prime}\right)} \\
& =\sum_{j=1}^{k} \int d q_{k+1} d q_{k+1}^{\prime} \\
& \quad \times\left\{\gamma^{(k+1)}\left(p_{1}, \ldots, p_{j}-q_{k+1}+q_{k+1}^{\prime}, \ldots, p_{k}, q_{k+1} ; \mathbf{p}_{k}^{\prime}, q_{k+1}^{\prime}\right)\right. \\
& \left.\quad \quad-\gamma^{(k+1)}\left(\mathbf{p}_{k}, q_{k+1} ; p_{1}^{\prime}, \ldots, p_{j}^{\prime}+q_{k+1}-q_{k+1}^{\prime}, \ldots, p_{k}^{\prime}, q_{k+1}^{\prime}\right)\right\} \\
& =\sum_{j=1}^{k} \int d \mathbf{q}_{k+1} d \mathbf{q}_{k+1}^{\prime}\left[\prod_{l \neq j}^{k} \delta\left(p_{l}-q_{l}\right) \delta\left(p_{l}^{\prime}-q_{l}^{\prime}\right)\right] \\
& \quad \times \gamma^{(k+1)}\left(\mathbf{q}_{k+1} ; \mathbf{q}_{k+1}^{\prime}\right)\left\{\delta\left(p_{j}^{\prime}-q_{j}^{\prime}\right) \delta\left(p_{j}-\left[q_{j}+q_{k+1}-q_{k+1}^{\prime}\right]\right)\right. \\
& \left.\quad-\delta\left(p_{j}-q_{j}\right) \delta\left(p_{j}^{\prime}-\left[q_{j}^{\prime}+q_{k+1}^{\prime}-q_{k+1}\right]\right)\right\} .
\end{aligned}
$$

We begin with the following simple lemma.

Lemma 3.1. If $\beta>n$, then

$$
\sup _{p \in \mathbb{R}^{n}} \int_{\mathbb{R}^{n}} \mathrm{~d} q \mathrm{~d} q^{\prime} \frac{\langle p\rangle^{\beta}}{\left\langle p+q^{\prime}-q\right\rangle^{\beta}\langle q\rangle^{\beta}\left\langle q^{\prime}\right\rangle^{\beta}}<\infty .
$$

Proof. Since

$$
\begin{aligned}
& \frac{1}{\left\langle p+q^{\prime}-q\right\rangle^{\beta}\langle q\rangle^{\beta}\left\langle q^{\prime}\right\rangle^{\beta}} \\
\leq & \frac{2^{\beta}}{\left\langle p+q^{\prime}\right\rangle^{\beta}\left\langle q^{\prime}\right\rangle^{\beta}}\left(\frac{1}{\left\langle p+q^{\prime}-q\right\rangle^{\beta}}+\frac{1}{\langle q\rangle^{\beta}}\right) \\
\leq & \frac{2^{2 \beta}}{\langle p\rangle^{\beta}}\left(\frac{1}{\left\langle p+q^{\prime}\right\rangle^{\beta}}+\frac{1}{\left\langle q^{\prime}\right\rangle^{\beta}}\right)\left(\frac{1}{\left\langle p+q^{\prime}-q\right\rangle^{\beta}}+\frac{1}{\langle q\rangle^{\beta}}\right),
\end{aligned}
$$

the inequality (3.2) is concluded from the assumption $\beta>n$.

As in [14], we introduce for $\gamma^{(k+1)}\left(\mathbf{x}_{k+1}, \mathbf{x}_{k+1}^{\prime}\right) \in \mathcal{S}\left(\mathbb{R}^{(k+1) n} \times \mathbb{R}^{(k+1) n}\right)$,

$$
\begin{aligned}
& {\left[B_{j, k}^{1} \gamma^{(k+1)}\right]\left(\mathbf{x}_{k}, \mathbf{x}_{k}^{\prime}\right)} \\
& \quad=\int \mathrm{d} x_{k+1} \mathrm{~d} x_{k+1}^{\prime} \delta\left(x_{k+1}-x_{k+1}^{\prime}\right) \delta\left(x_{j}-x_{k+1}\right) \gamma^{(k+1)}\left(\mathbf{x}_{k+1}, \mathbf{x}_{k+1}^{\prime}\right),
\end{aligned}
$$

and

$$
\begin{aligned}
& {\left[B_{j, k}^{2} \gamma^{(k+1)}\right]\left(\mathbf{x}_{k}, \mathbf{x}_{k}^{\prime}\right)} \\
& \quad=\int \mathrm{d} x_{k+1} \mathrm{~d} x_{k+1}^{\prime} \delta\left(x_{k+1}-x_{k+1}^{\prime}\right) \delta\left(x_{j}^{\prime}-x_{k+1}\right) \gamma^{(k+1)}\left(\mathbf{x}_{k+1}, \mathbf{x}_{k+1}^{\prime}\right),
\end{aligned}
$$


where $j=1, \ldots, k$. Then, by (2.4) we have

$$
B^{(k)}=\sum_{j=1}^{k}\left(B_{j, k}^{1}-B_{j, k}^{2}\right)
$$

acting on smooth kernel functions $\gamma^{(k+1)} \in \mathcal{S}\left(\mathbb{R}^{(k+1) n} \times \mathbb{R}^{(k+1) n}\right)$.

The following estimate is crucial for the proof of Theorem 2.1. In fact, the proof of the lemma is completely analogous to that of $\|f\|_{L^{\infty}\left(\mathbb{R}^{n}\right)} \leq$ $C\|f\|_{H^{\alpha}\left(\mathbb{R}^{n}\right)}$ based on Fourier analysis.

Lemma 3.2. Suppose that $\alpha>\frac{n}{2}$ and $n \geq 1$. Then, there exists a constant $C_{\alpha, n}>0$ depending only on $\alpha$ and $n$ such that, for any $\gamma^{(k+1)} \in \mathcal{S}\left(\mathbb{R}^{(k+1) n} \times\right.$ $\left.\mathbb{R}^{(k+1) n}\right)$,

$$
\left\|B_{j, k}^{l} \gamma^{(k+1)}\right\|_{\mathrm{H}_{k}^{\alpha}} \leq C_{\alpha, n}\left\|\gamma^{(k+1)}\right\|_{\mathrm{H}_{k+1}^{\alpha}}, \quad l=1,2,
$$

for all $k \geq 1$, where $j=1, \cdots, k$. Consequently,

$$
\left\|B^{(k)} \gamma^{(k+1)}\right\|_{\mathrm{H}_{k}^{\alpha}} \leq C_{\alpha, n} k\left\|\gamma^{(k+1)}\right\|_{\mathrm{H}_{k+1}^{\alpha}}
$$

for any $\gamma^{(k+1)} \in \mathcal{S}\left(\mathbb{R}^{(k+1) n} \times \mathbb{R}^{(k+1) n}\right)$ and all $k \geq 1$.

Remark 3.1. The estimate (3.3) indicates that the operator $B^{(k)}$, originally defined on Schwarz functions, can be extended to a bounded operator from $\mathrm{H}_{k+1}^{\alpha}$ to $\mathrm{H}_{k}^{\alpha}$. In this case, we still denote it by $B^{(k)}$.

Proof. We first consider $B_{1, k}^{1}$. For $\gamma^{(k+1)} \in \mathcal{S}\left(\mathbb{R}^{(k+1) n} \times \mathbb{R}^{(k+1) n}\right)$, from the Plancherel's theorem it is concluded that

$$
\begin{aligned}
\left\|B_{1, k}^{1} \gamma^{(k+1)}\right\|_{\mathrm{H}_{k}^{\alpha}}^{2}= & \int \prod_{j=1}^{k}\left\langle p_{j}\right\rangle^{2 \alpha}\left\langle p_{j}^{\prime}\right\rangle^{2 \alpha} \mathrm{d} \mathbf{p}_{k} \mathrm{~d} \mathbf{p}_{k}^{\prime} \mid \int \mathrm{d} q \mathrm{~d} q^{\prime} \\
& \left.\gamma^{(k+1)}\left(p_{1}+q^{\prime}-q, p_{2}, \cdots, p_{k}, q ; p_{1}^{\prime}, \cdots, p_{k}^{\prime}, q^{\prime}\right)\right|^{2} .
\end{aligned}
$$


Then, we obtain

$$
\begin{aligned}
\left\|B_{1, k}^{1} \gamma^{(k+1)}\right\|_{\mathrm{H}_{k}^{\alpha}}^{2} & \\
\leq & \int \prod_{j=1}^{k}\left\langle p_{j}\right\rangle^{2 \alpha}\left\langle p_{j}^{\prime}\right\rangle^{2 \alpha} \mathrm{d} \mathbf{p}_{k} \mathrm{~d} \mathbf{p}_{k}^{\prime} \\
& \times\left(\int \mathrm{d} q \mathrm{~d} q^{\prime} \frac{1}{\left\langle p_{1}+q^{\prime}-q\right\rangle^{2 \alpha}\langle q\rangle^{2 \alpha}\left\langle q^{\prime}\right\rangle^{2 \alpha}}\right) \\
& \times\left(\int \mathrm{d} q \mathrm{~d} q^{\prime}\left\langle p_{1}+q^{\prime}-q\right\rangle^{2 \alpha}\langle q\rangle^{2 \alpha}\left\langle q^{\prime}\right\rangle^{2 \alpha}\right. \\
& \left.\times\left|\gamma^{(k+1)}\left(p_{1}+q^{\prime}-q, p_{2}, \cdots, p_{k}, q ; p_{1}^{\prime}, \cdots, p_{k}^{\prime}, q^{\prime}\right)\right|^{2}\right) \\
\leq & \sup _{p_{1} \in \mathbb{R}^{n}} \int \mathrm{d} q \mathrm{~d} q^{\prime} \frac{\left\langle p_{1}\right\rangle^{2 \alpha}}{\left\langle p_{1}+q^{\prime}-q\right\rangle^{2 \alpha}\langle q\rangle^{2 \alpha}\left\langle q^{\prime}\right\rangle^{2 \alpha}} \\
& \times \int \prod_{j=2}^{k}\left\langle p_{j}\right\rangle^{2 \alpha}\left\langle p_{j}^{\prime}\right\rangle^{2 \alpha} \mathrm{d} \mathbf{p}_{k} \mathrm{~d} \mathbf{p}_{k}^{\prime} \mathrm{d} q \mathrm{~d} q^{\prime} \\
& \times\left\{\left\langle p_{1}+q^{\prime}-q\right\rangle^{2 \alpha}\left\langle p_{1}^{\prime}\right\rangle^{2 \alpha}\langle q\rangle^{2 \alpha}\left\langle q^{\prime}\right\rangle^{2 \alpha}\right. \\
& \left.\times\left|\gamma^{(k+1)}\left(p_{1}+q^{\prime}-q, p_{2}, \cdots, p_{k}, q ; p_{1}^{\prime}, \cdots, p_{k}^{\prime}, q^{\prime}\right)\right|^{2}\right\} \\
= & \sup _{p_{1} \in \mathbb{R}^{n}} \int \mathrm{d} q \mathrm{~d} q^{\prime} \frac{\left\langle p_{1}\right\rangle^{2 \alpha}}{\left\langle p_{1}+q^{\prime}-q\right\rangle^{2 \alpha}\langle q\rangle^{2 \alpha}\left\langle q^{\prime}\right\rangle^{2 \alpha}}\left\|\gamma^{(k+1)}\right\|_{\mathrm{H}_{k+1}^{\alpha}}^{2} \\
\leq & C_{\alpha, n}\left\|\gamma^{(k+1)}\right\|_{\mathrm{H}_{k+1}^{\alpha}}^{2},
\end{aligned}
$$

where we have used Lemma 3.1 in the last inequality. For the operator $B_{1, k}^{2}$, we have the same estimate

$$
\left\|B_{1, k}^{2} \gamma^{(k+1)}\right\|_{\mathrm{H}_{k}^{\alpha}} \leq C_{\alpha, n}\left\|\gamma^{(k+1)}\right\|_{\mathrm{H}_{k+1}^{\alpha}} .
$$

Similarly, we can prove the same bound for $B_{j, k}^{1}$ and $B_{j, k}^{2}$ when $j=2, \cdots, k$. Consequently, we conclude the estimate (3.3).

\section{Proof of Theorem 2.1}

Now we are ready to prove Theorem 2.1. The proof is divided into two parts as follows.

Proof. (i) Let $\alpha>n / 2$ and $0<\xi<1$. Given $\Gamma_{0}=\left\{\gamma_{0}^{(k)}\right\}_{k \geq 1} \in \mathcal{H}_{\xi}^{\alpha}$. For $m \geq 1$, set

$$
\gamma_{m, t}^{(k)}=\mathcal{U}_{0}^{(k)}(t) \gamma_{0}^{(k)}+\int_{0}^{t} \mathrm{~d} s \mathcal{U}_{0}^{(k)}(t-s) \tilde{B}^{(k)} \gamma_{m-1, s}^{(k+1)}, \quad t>0, k \geq 1,
$$


with the convention $\gamma_{0, t}^{(k)} \equiv \gamma_{0}^{(k)}$, where $\tilde{B}^{(k)}=-i \mu B^{(k)}$ (e.g., (2.6)). By expansion, for every $m \geq 1$ one has

$$
\begin{aligned}
\gamma_{m, t}^{(k)}= & \mathcal{U}_{0}^{(k)}(t) \gamma_{0}^{(k)}+\sum_{j=1}^{m-1} \int_{0}^{t} \mathrm{~d} t_{1} \int_{0}^{t_{1}} \mathrm{~d} t_{2} \cdots \int_{0}^{t_{j-1}} \mathrm{~d} t_{j} \mathcal{U}_{0}^{(k)}\left(t-t_{1}\right) \tilde{B}^{(k)} \ldots \\
& \times \mathcal{U}_{0}^{(k+j-1)}\left(t_{j-1}-t_{j}\right) \tilde{B}^{(k+j-1)} \mathcal{U}_{0}^{(k+j)}\left(t_{j}\right) \gamma_{0}^{(k+j)} \\
& +\int_{0}^{t} \mathrm{~d} t_{1} \int_{0}^{t_{1}} \mathrm{~d} t_{2} \cdots \int_{0}^{t_{m-1}} \mathrm{~d} t_{m} \mathcal{U}_{0}^{(k)}\left(t-t_{1}\right) \tilde{B}^{(k)} \ldots \\
& \times \mathcal{U}_{0}^{(k+m-1)}\left(t_{m-1}-t_{m}\right) \tilde{B}^{(k+m-1)} \gamma_{0}^{(k+m)} \\
\triangleq & \sum_{j=0}^{m} \Xi_{j, t}^{(k)}
\end{aligned}
$$

with the convention $t_{0}=t$. Then, for $j=1, \cdots, m-1$, by Lemma 3.2 we have

$$
\begin{aligned}
\left\|\Xi_{j, t}^{(k)}\right\|_{\mathrm{H}_{k}^{\alpha}} \leq & \int_{0}^{t} \mathrm{~d} t_{1} \int_{0}^{t_{1}} \mathrm{~d} t_{2} \cdots \int_{0}^{t_{j-1}} \mathrm{~d} t_{j} \| \mathcal{U}_{0}^{(k)}\left(t-t_{1}\right) \tilde{B}^{(k)} \ldots \\
& \times \mathcal{U}_{0}^{(k+j-1)}\left(t_{j-1}-t_{j}\right) \tilde{B}^{(k+j-1)} \mathcal{U}_{0}^{(k+j)}\left(t_{j}\right) \gamma_{0}^{(k+j)} \|_{\mathrm{H}_{k}^{\alpha}} \\
\leq & \int_{0}^{t} \mathrm{~d} t_{1} \int_{0}^{t_{1}} \mathrm{~d} t_{2} \cdots \int_{0}^{t_{j-1}} \mathrm{~d} t_{j} k \cdots \\
& \times(k+j-1)\left(C_{\alpha, n}\right)^{j}\left\|\mathcal{U}_{0}^{(k+j)}\left(t_{j}\right) \gamma_{0}^{(k+j)}\right\|_{\mathrm{H}_{k+j}^{\alpha}} \\
= & \frac{t^{j}}{j !} \cdots(k+j-1)\left(C_{\alpha, n}\right)^{j}\left\|\gamma_{0}^{(k+j)}\right\|_{\mathrm{H}_{k+j}^{\alpha}} \\
= & \left(\begin{array}{c}
k+j-1 \\
j
\end{array}\right)\left(C_{\alpha, n} t\right)^{j}\left\|\gamma_{0}^{(k+j)}\right\|_{\mathrm{H}_{k+j}^{\alpha}},
\end{aligned}
$$

and

$$
\begin{aligned}
\left\|\Xi_{m, t}^{(k)}\right\|_{\mathrm{H}_{k}^{\alpha}} \leq & \int_{0}^{t} \mathrm{~d} t_{1} \int_{0}^{t_{1}} \mathrm{~d} t_{2} \cdots \int_{0}^{t_{m-1}} \mathrm{~d} t_{m} \| \mathcal{U}_{0}^{(k)}\left(t-t_{1}\right) \tilde{B}^{(k)} \ldots \\
& \times \mathcal{U}_{0}^{(k+m-1)}\left(t_{m-1}-t_{m}\right) \tilde{B}^{(k+m-1)} \gamma_{0}^{(k+m)} \|_{\mathrm{H}_{k}^{\alpha}} \\
\leq & \int_{0}^{t} \mathrm{~d} t_{1} \int_{0}^{t_{1}} \mathrm{~d} t_{2} \cdots \int_{0}^{t_{m-1}} \mathrm{~d} t_{m} k \cdots \\
& \times(k+m-1)\left(C_{\alpha, n}\right)^{m}\left\|\gamma_{0}^{(k+m)}\right\|_{\mathrm{H}_{k+m}^{\alpha}} \\
\leq & \frac{t^{m}}{m !} k \cdots(k+m-1)\left(C_{\alpha, n}\right)^{m}\left\|\gamma_{0}^{(k+m)}\right\|_{\mathrm{H}_{k+m}^{\alpha}} \\
= & \left(\begin{array}{c}
k+m-1 \\
m
\end{array}\right)\left(C_{\alpha, n} t\right)^{m}\left\|\gamma_{0}^{(k+m)}\right\|_{\mathrm{H}_{k+m}^{\alpha}} .
\end{aligned}
$$


Then, for $T>0$ ( $T$ will be fixed in the sequel) we obtain

$$
\begin{aligned}
\left\|\gamma_{m, t}^{(k)}\right\|_{C\left([0, T], \mathrm{H}_{k}^{\alpha}\right)} & \leq \sum_{j=0}^{m}\left\|\Xi_{j}^{(k)}\right\|_{C\left([0, T], \mathrm{H}_{k}^{\alpha}\right)} \\
& \leq \sum_{j=0}^{m}\left(\begin{array}{c}
k+j-1 \\
j
\end{array}\right)\left(C_{\alpha, n} T\right)^{j}\left\|\gamma_{0}^{(k+j)}\right\|_{\mathrm{H}_{k+j}^{\alpha}}
\end{aligned}
$$

Hence, for $0<\eta<1$ (which will be fixed later) one has

$$
\begin{aligned}
& \sum_{k=1}^{\infty} \eta^{k}\left\|\gamma_{m, t}^{(k)}\right\|_{C\left([0, T], \mathrm{H}_{k}^{\alpha}\right)} \\
& \quad \leq \sum_{j=0}^{m} \sum_{k=1}^{\infty} \eta^{k}\left(\begin{array}{c}
k+j-1 \\
j
\end{array}\right)\left(C_{\alpha, n} T\right)^{j}\left\|\gamma_{0}^{(k+j)}\right\|_{\mathrm{H}_{k+j}^{\alpha}} \\
& \quad \leq \sum_{j=0}^{\infty} \sum_{k=1}^{\infty} \eta^{k}\left(\begin{array}{c}
k+j-1 \\
j
\end{array}\right)\left(C_{\alpha, n} T\right)^{j}\left\|\gamma_{0}^{(k+j)}\right\|_{\mathrm{H}_{k+j}^{\alpha}}
\end{aligned}
$$

By the direct computation, one has

$$
\begin{aligned}
\sum_{j=0}^{\infty} \sum_{k=1}^{\infty} \eta^{k}\left(\begin{array}{c}
k+j-1 \\
j
\end{array}\right)\left(C_{\alpha, n} T\right)^{j}\left\|\gamma_{0}^{(k+j)}\right\|_{\mathrm{H}_{k+j}^{\alpha}} \\
=\sum_{j=0}^{\infty} \sum_{l=j+1}^{\infty} \eta^{l-j}\left(\begin{array}{c}
l-1 \\
j
\end{array}\right)\left(C_{\alpha, n} T\right)^{j}\left\|\gamma_{0}^{(l)}\right\|_{\mathrm{H}_{l}^{\alpha}} \\
=\sum_{l=1}^{\infty} \sum_{j=0}^{l-1}\left(\begin{array}{c}
l-1 \\
j
\end{array}\right)\left(C_{\alpha, n} T / \eta\right)^{j} \eta^{l}\left\|\gamma_{0}^{(l)}\right\|_{\mathrm{H}_{l}^{\alpha}} \\
=\sum_{l=1}^{\infty}\left(1+C_{\alpha, n} T / \eta\right)^{l-1} \eta^{l}\left\|\gamma_{0}^{(l)}\right\|_{\mathrm{H}_{l}^{\alpha}} \\
=\frac{\eta}{\eta+C_{\alpha, n} T} \sum_{l=1}^{\infty}\left(\eta+C_{\alpha, n} T\right)^{l}\left\|\gamma_{0}^{(l)}\right\|_{\mathrm{H}_{l}^{\alpha} .}
\end{aligned}
$$

Set $\Gamma_{m, t}=\left\{\gamma_{m, t}^{(k)}\right\}$. Let $\eta=\xi-C_{\alpha, n} T$ with $0<T<\xi / C_{\alpha, n}$. Then, it follows from (4.3) that

$$
\left\|\Gamma_{m, t}\right\|_{C\left([0, T], \mathcal{H}_{\eta}^{\alpha}\right)} \leq \frac{\eta}{\xi} \sum_{l=1}^{\infty} \xi^{l}\left\|\gamma_{0}^{(l)}\right\|_{\mathrm{H}_{l}^{\alpha}}=\frac{\eta}{\xi}\left\|\Gamma_{0}\right\|_{\mathcal{H}_{\xi}^{\alpha}} .
$$

Next, we prove that $\left\{\Gamma_{m, t}\right\}_{m \geq 1}$ converges to a solution. Indeed, by the above estimates for $\Xi_{j, t}^{(k)}$ we have for any $m, n$ with $n>m$

$$
\left\|\gamma_{m, t}^{(k)}-\gamma_{n, t}^{(k)}\right\|_{C\left([0, T], \mathrm{H}_{k}^{\alpha}\right)} \leq 2 \sum_{j=m}^{n}\left(\begin{array}{c}
k+j-1 \\
j
\end{array}\right)\left(C_{\alpha, n} T\right)^{j}\left\|\gamma_{0}^{(k+j)}\right\|_{\mathrm{H}_{k+j}^{\alpha}}
$$


Then,

$$
\left\|\Gamma_{m, t}-\Gamma_{n, t}\right\|_{C_{t \in[0, T]} \mathcal{H}_{\eta}^{\alpha}} \leq 2 \sum_{j=m}^{n} \sum_{k=1}^{\infty} \eta^{k}\left(\begin{array}{c}
k+j-1 \\
j
\end{array}\right)\left(C_{\alpha, n} T\right)^{j}\left\|\gamma_{0}^{(k+j)}\right\|_{\mathrm{H}_{k+j}^{\alpha}} .
$$

An immediate computation as above yields that

$$
\left\|\Gamma_{m, t}-\Gamma_{n, t}\right\|_{C\left([0, T], \mathcal{H}_{\eta}^{\alpha}\right)} \leq 2 \frac{\eta}{\xi} \sum_{l=m+1}^{\infty} \xi^{l}\left\|\gamma_{0}^{(l)}\right\|_{\mathrm{H}_{l}^{\alpha}} .
$$

Since $\Gamma_{0}=\left\{\gamma_{0}^{(k)}\right\}_{k \geq 1} \in \mathcal{H}_{\xi}^{\alpha}$, it is concluded that $\left\{\Gamma_{m, t}\right\}_{m \geq 1}$ is a Cauchy sequence in $C\left([0, T], \mathcal{H}_{\eta}^{\alpha}\right)$ and so converges to some $\Gamma_{t} \in C\left([0, T], \mathcal{H}_{\eta}^{\alpha}\right)$. Taking the limit $m \rightarrow \infty$ in (4.1) we prove that $\Gamma_{t}$ is a solution to (2.2). Also, taking $m \rightarrow \infty$ in (4.4) yields this solution satisfies (2.10).

(ii) Choose $T=\xi /(5 C)$ and suppose $\Gamma_{t}, \Gamma_{t}^{\prime} \in C\left([0, T], \mathcal{H}_{\eta}^{\alpha}\right)$ are two solutions to $(2.2)$ with the initial datum $\Gamma_{0}$ and $\Gamma_{0}^{\prime}$ in $\mathcal{H}_{\xi}^{\alpha}$, respectively. Since (2.2) is linear, it suffices to consider $\Gamma_{t}$ instead of $\Gamma_{t}-\Gamma_{t}^{\prime}$. By (2.8), for every $m \geq 1$ one has

$$
\begin{aligned}
\gamma_{t}^{(k)}= & \mathcal{U}_{0}^{(k)}(t) \gamma_{0}^{(k)}+\sum_{j=1}^{m-1} \int_{0}^{t} \mathrm{~d} t_{1} \int_{0}^{t_{1}} \mathrm{~d} t_{2} \cdots \int_{0}^{t_{j-1}} \mathrm{~d} t_{j} \mathcal{U}_{0}^{(k)}\left(t-t_{1}\right) \tilde{B}^{(k)} \ldots \\
& \times \mathcal{U}_{0}^{(k+j-1)}\left(t_{j-1}-t_{j}\right) \tilde{B}^{(k+j-1)} \mathcal{U}_{0}^{(k+j)}\left(t_{j}\right) \gamma_{0}^{(k+j)} \\
& +\int_{0}^{t} \mathrm{~d} t_{1} \int_{0}^{t_{1}} \mathrm{~d} t_{2} \cdots \int_{0}^{t_{m-1}} \mathrm{~d} t_{m} \mathcal{U}_{0}^{(k)}\left(t-t_{1}\right) \tilde{B}^{(k)} \ldots \\
& \times \mathcal{U}_{0}^{(k+m-1)}\left(t_{m-1}-t_{m}\right) \tilde{B}^{(k+m-1)} \gamma_{t_{m}}^{(k+m)} \\
\triangleq & \sum_{j=0}^{m-1} \Xi_{j, t}^{(k)}+\tilde{\Xi}_{m, t}^{(k)}
\end{aligned}
$$

with the convention $t_{0}=t$. Note that,

$$
\begin{aligned}
\left\|\tilde{\Xi}_{m, t}^{(k)}\right\|_{\mathrm{H}_{k}^{\alpha} \leq} \leq & \int_{0}^{t} \mathrm{~d} t_{1} \int_{0}^{t_{1}} \mathrm{~d} t_{2} \cdots \int_{0}^{t_{m-1}} \mathrm{~d} t_{m} \| \mathcal{U}_{0}^{(k)}\left(t-t_{1}\right) \tilde{B}^{(k)} \ldots \\
& \times \mathcal{U}_{0}^{(k+m-1)}\left(t_{m-1}-t_{m}\right) \tilde{B}^{(k+m-1)} \gamma_{t_{m}}^{(k+m)} \|_{\mathrm{H}_{k}^{\alpha}} \\
\leq & \int_{0}^{t} \mathrm{~d} t_{1} \int_{0}^{t_{1}} \mathrm{~d} t_{2} \cdots \int_{0}^{t_{m-1}} \mathrm{~d} t_{m} k \cdots \\
& \quad \times(k+m-1)\left(C_{\alpha, n}\right)^{m}\left\|\gamma_{t_{m}}^{(k+m)}\right\|_{\mathrm{H}_{k+m}^{\alpha}} \\
\leq & m(m+1) \cdots(2 m-1)\left(C_{\alpha, n}\right)^{m} \\
& \quad \times \int_{0}^{t} \mathrm{~d} t_{1} \int_{0}^{t_{1}} \mathrm{~d} t_{2} \cdots \int_{0}^{t_{m-1}}\left\|\gamma_{t_{m}}^{(k+m)}\right\|_{\mathrm{H}_{k+m}^{\alpha}} \mathrm{d} t_{m} .
\end{aligned}
$$


Combining this estimate and (4.2) yields

$$
\begin{aligned}
\left\|\gamma_{t}^{(k)}\right\|_{\mathrm{H}_{k}^{\alpha} \leq} \leq & \sum_{j=0}^{m-1}\left(\begin{array}{c}
k+j-1 \\
j
\end{array}\right)\left(C_{\alpha, n} T\right)^{j}\left\|\gamma_{0}^{(k+j)}\right\|_{\mathrm{H}_{k+j}^{\alpha}} \\
& +m(m+1) \cdots(2 m-1)\left(C_{\alpha, n}\right)^{m} \\
& \times \int_{0}^{t} \mathrm{~d} t_{1} \int_{0}^{t_{1}} \mathrm{~d} t_{2} \cdots \int_{0}^{t_{m-1}}\left\|\gamma_{t_{m}}^{(k+m)}\right\|_{\mathrm{H}_{k+m}^{\alpha}} \mathrm{d} t_{m} .
\end{aligned}
$$

Then for $m \geq 1$ and $0<t<T$, we have

$$
\begin{aligned}
\sum_{k=1}^{m} \eta^{k}\left\|\gamma_{t}^{(k)}\right\|_{\mathrm{H}_{k}^{\alpha}} & \sum_{j=0}^{m-1} \sum_{k=1}^{m}\left(\begin{array}{c}
k+j-1 \\
j
\end{array}\right)\left(C_{\alpha, n} T / \eta\right)^{j} \eta^{k+j}\left\|\gamma_{0}^{(k+j)}\right\|_{\mathrm{H}_{k+j}^{\alpha}} \\
& +m(m+1) \cdots(2 m-1)\left(\frac{C_{\alpha, n}}{\eta}\right)^{m} \\
& \times \int_{0}^{t} \mathrm{~d} t_{1} \int_{0}^{t_{1}} \mathrm{~d} t_{2} \cdots \int_{0}^{t_{m-1}} \sum_{k=1}^{m} \eta^{(k+m)}\left\|\gamma_{t_{m}}^{(k+m)}\right\|_{\mathrm{H}_{k+m}^{\alpha}} \mathrm{d} t_{m} \\
\leq & \frac{\eta}{\xi}\left\|\Gamma_{0}\right\|_{\mathcal{H}_{\xi}^{\alpha}}+\left(\frac{C_{\alpha, n} t}{\eta}\right)^{m}\left(\begin{array}{c}
2 m-1 \\
m
\end{array}\right)\left\|\Gamma_{t}\right\|_{C\left([0, T], \mathcal{H}_{\eta}^{\alpha}\right)} \\
\leq & \frac{4}{5}\left\|\Gamma_{0}\right\|_{\mathcal{H}_{\xi}^{\alpha}}+\left(\begin{array}{c}
2 m-1 \\
m
\end{array}\right)\left(\frac{C_{\alpha, n} T}{\eta}\right)^{m}\left\|\Gamma_{t}\right\|_{C\left([0, T], \mathcal{H}_{\eta}^{\alpha}\right)} .
\end{aligned}
$$

By Stirling's formula $m ! \approx m^{m+1 / 2} e^{-m}$ we have

$$
\left(\begin{array}{c}
2 m-1 \\
m
\end{array}\right) \approx \frac{4^{m}}{\sqrt{m}}
$$

Then, taking $m \rightarrow \infty$ in (4.5) yields that

$$
\left\|\Gamma_{t}\right\|_{C\left([0, T], \mathcal{H}_{\eta}^{\alpha}\right)} \leq \frac{4}{5}\left\|\Gamma_{0}\right\|_{\mathcal{H}_{\xi}^{\alpha}}
$$

because $C_{\alpha, n} T / \eta=1 / 4$. Thus, the space-time type bound (2.11) holds true.

The uniqueness of the solution in $C\left([0, T], \mathcal{H}_{\eta}^{\alpha}\right)$ follows clearly from the estimate (2.11).

\section{The quintic Gross-Pitaevskit hierarchy}

In this section, we consider the so-called quintic Gross-Pitaevskii hierarchy. Recall that the quintic Gross-Pitaevskii hierarchy is given by

$$
i \partial_{t} \gamma_{t}^{(k)}=\left[-\Delta^{(k)}, \gamma_{t}^{(k)}\right]+\mu Q^{(k)} \gamma_{t}^{(k+2)}, \Delta^{(k)}=\sum_{j=1}^{k} \Delta_{x_{j}}, \mu= \pm 1
$$


in $n$ dimensions, for $k \in \mathbb{N}$, where the operator $Q^{(k)}$ is defined by

$$
Q^{(k)} \gamma_{t}^{(k+2)}=\sum_{j=1}^{k} \operatorname{tr}_{k+1, k+2}\left[\delta\left(x_{j}-x_{k+1}\right) \delta\left(x_{j}-x_{k+2}\right), \gamma_{t}^{(k+2)}\right]
$$

It is defocusing if $\mu=1$, and focusing if $\mu=-1$. We note that the quintic Gross-Pitaevskii hierarchy accounts for 3-body interactions between the Bose particles (see [4] and references therein for details).

Remark 5.1. In terms of kernel functions we can rewrite (5.1) as follows

$$
\left(i \partial_{t}+\triangle_{ \pm}^{(k)}\right) \gamma_{t}^{(k)}\left(\mathbf{x}_{k} ; \mathbf{x}_{k}^{\prime}\right)=\mu\left(Q^{(k)} \gamma_{t}^{(k+2)}\right)\left(\mathbf{x}_{k} ; \mathbf{x}_{k}^{\prime}\right)
$$

where, the action of $Q^{(k)}$ on $\gamma^{(k+2)}\left(\mathbf{x}_{k+2}, \mathbf{x}_{k+2}^{\prime}\right) \in \mathcal{S}\left(\mathbb{R}^{(k+2) n} \times \mathbb{R}^{(k+2) n}\right)$ is given by

$$
\begin{aligned}
\left(Q^{(k)} \gamma^{(k+2)}\right)\left(\mathbf{x}_{k}, \mathbf{x}_{k}^{\prime}\right) \\
:=\sum_{j=1}^{k}\left(Q_{j, k} \gamma^{(k+2)}\right)\left(\mathbf{x}_{k}, \mathbf{x}_{k}^{\prime}\right) \\
:=\sum_{j=1}^{k} \int d x_{k+1} d x_{k+2} d x_{k+1}^{\prime} d x_{k+2}^{\prime} \gamma^{(k+2)}\left(\mathbf{x}_{k}, x_{k+1}, x_{k+2} ; \mathbf{x}_{k}^{\prime}, x_{k+1}^{\prime}, x_{k+2}^{\prime}\right) \\
\quad \times\left[\prod_{\ell=k+1}^{k+2} \delta\left(x_{j}-x_{\ell}\right) \delta\left(x_{j}-x_{\ell}^{\prime}\right)-\prod_{\ell=k+1}^{k+2} \delta\left(x_{j}^{\prime}-x_{\ell}\right) \delta\left(x_{j}^{\prime}-x_{\ell}^{\prime}\right)\right] .
\end{aligned}
$$

Let $\varphi \in \mathrm{H}^{1}\left(\mathbb{R}^{n}\right)$, then one can easily verify that a particular solution to (5.2) with initial conditions

$$
\gamma_{t=0}^{(k)}\left(\mathbf{x}_{k} ; \mathbf{x}_{k}^{\prime}\right)=\prod_{j=1}^{k} \varphi\left(x_{j}\right) \overline{\varphi\left(x_{j}^{\prime}\right)}, \quad k=1,2, \ldots
$$

is given by

$$
\gamma_{t}^{(k)}\left(\mathbf{x}_{k} ; \mathbf{x}_{k}^{\prime}\right)=\prod_{j=1}^{k} \varphi_{t}\left(x_{j} \overline{\varphi_{t}\left(x_{j}^{\prime}\right)} k=1,2, \ldots,\right.
$$

where $\varphi_{t}$ satisfies the quintic non-linear Schrödinger equation

$$
i \partial_{t} \varphi_{t}=-\Delta \varphi_{t}+\mu\left|\varphi_{t}\right|^{4} \varphi_{t}, \quad \varphi_{t=0}=\varphi .
$$

Remark 5.2. The Gross-Pitaevskii hierarchy (5.1) can be written in the integral form

$$
\gamma_{t}^{(k)}=\mathcal{U}_{0}^{(k)}(t) \gamma_{0}^{(k)}+\int_{0}^{t} d s \mathcal{U}_{0}^{(k)}(t-s) \tilde{Q}^{(k)} \gamma_{s}^{(k+2)}, k=1,2, \ldots
$$


where $\tilde{Q}^{(k)}=-i \mu Q^{(k)}$. Formally we can expand the solution $\gamma_{t}^{(k)}$ of (5.4) for any $m \geq 1$ as

$$
\begin{aligned}
\gamma_{t}^{(k)} & =\mathcal{U}_{0}^{(k)}(t) \gamma_{0}^{(k)}+\sum_{j=1}^{m-1} \int_{0}^{t} d s_{1} \int_{0}^{s_{1}} d s_{2} \cdots \int_{0}^{s_{j-1}} d s_{j} \mathcal{U}_{0}^{(k)}\left(t-s_{1}\right) \tilde{Q}^{(k)} \ldots \\
& \times \mathcal{U}_{0}^{(k+j-1)}\left(s_{j-1}-s_{j}\right) \tilde{Q}^{(k+j-1)} \mathcal{U}_{0}^{(k+2+j)}\left(s_{j}\right) \gamma_{0}^{(k+2+j)} \\
& +\int_{0}^{t} d s_{1} \int_{0}^{s_{1}} d s_{2} \cdots \int_{0}^{s_{m-1}} d s_{m} \mathcal{U}_{0}^{(k)}\left(t-s_{1}\right) \tilde{Q}^{(k)} \ldots \\
& \times \mathcal{U}_{0}^{(k+m-1)}\left(s_{m-1}-s_{m}\right) \tilde{Q}^{(k+m-1)} \gamma_{s_{m}}^{(k+2+m)}
\end{aligned}
$$

with the convention $s_{0}=t$.

Let $\mathbf{q}=\left(p_{k+1}, p_{k+2}\right)$ and $\mathbf{q}^{\prime}=\left(p_{k+1}^{\prime}, p_{k+2}^{\prime}\right)$ we have

$$
\begin{aligned}
& \left(Q_{j, k} \gamma^{(k+2)}\right)\left(\mathbf{p}_{k}, \mathbf{p}_{k}^{\prime}\right) \\
= & \int d \mathbf{q} d \mathbf{q}^{\prime}\left[\gamma^{(k+2)}\left(p_{1}, \ldots, p_{j}+p_{k+1}+p_{k+2}-p_{k+1}^{\prime}-p_{k+2}^{\prime}, \ldots, p_{k}, \mathbf{q} ; \mathbf{p}_{k+2}^{\prime}\right)\right. \\
& \left.-\gamma^{(k+2)}\left(\mathbf{p}_{k+2} ; p_{1}^{\prime}, \ldots, p_{j}^{\prime}+p_{k+1}^{\prime}+p_{k+2}^{\prime}-p_{k+1}-p_{k+2}, \ldots, p_{k}^{\prime}, \mathbf{q}^{\prime}\right)\right]
\end{aligned}
$$

It is proved in [4] (Theorem 4.3 there) that for $\alpha>n / 2$ there exists a constant $C=C_{n, \alpha}>0$ depending only on $n$ and $\alpha$ such that

$$
\left\|Q_{j, k} \gamma^{(k+2)}\right\|_{\mathrm{H}_{k}^{\alpha}} \leq C\left\|\gamma^{(k+2)}\right\|_{\mathrm{H}_{k+2}^{\alpha}}, \quad \forall j=1, \ldots, k .
$$

Then, by slightly repeating the proof of Theorem 2.1, we can obtain the following theorem.

Theorem 5.1. Assume that $n \geq 1$ and $\alpha>n / 2$. Suppose $\Gamma_{0}=\left\{\gamma_{0}^{(k)}\right\}_{k \geq 1} \in$ $\mathcal{H}_{\xi}^{\alpha}$ for some $0<\xi<1$. Then there exists a constant $C=C_{\alpha, n}$ depending only on $n$ and $\alpha$ such that, for a fixed $0<T<\xi / C$ with $\eta=\xi-C T$, the following hold.

(i) There exists a solution $\Gamma_{t}=\left\{\gamma_{t}^{(k)}\right\}_{k \geq 1} \in C\left([0, T], \mathcal{H}_{\eta}^{\alpha}\right)$ to the GrossPitaevskii hierarchy (5.1) with the initial data $\Gamma_{0}$ satisfying

$$
\left\|\Gamma_{t}\right\|_{C\left([0, T], \mathcal{H}_{\eta}^{\alpha}\right)} \leq \frac{1}{\eta \xi}\left\|\Gamma_{0}\right\|_{\mathcal{H}_{\xi}^{\alpha}} .
$$

(ii) For $T=\xi /(5 C)$, if $\Gamma_{t}$ and $\Gamma_{t}^{\prime}$ in $C\left([0, T], \mathcal{H}_{\eta}^{\alpha}\right)$ are two solutions to (5.1) with initial conditions $\Gamma_{t=0}=\Gamma_{0}$ and $\Gamma_{t=0}^{\prime}=\Gamma_{0}^{\prime}$ in $\mathcal{H}_{\xi}^{\alpha}$ respectively, then

$$
\left\|\Gamma_{t}-\Gamma_{t}^{\prime}\right\|_{C\left([0, T], \mathcal{H}_{\eta}^{\alpha}\right)} \leq \frac{5}{4 \xi^{2}}\left\|\Gamma_{0}-\Gamma_{0}^{\prime}\right\|_{\mathcal{H}_{\xi}^{\alpha}} .
$$

Consequently, the solution $\Gamma_{t}$ to the initial problem (5.1) with the initial data in $\mathcal{H}_{\xi}^{\alpha}$ is unique in $C\left([0, T], \mathcal{H}_{\eta}^{\alpha}\right)$ for any $0<T<\xi / C$. 
We omit the details of the proof. This result also improves the corresponding one in [2] for the regime $\alpha>\frac{n}{2}$.

Acknowledgment We are grateful to the anonymous referee for many helpful comments and suggestions, which have been incorporated into this version of the paper.

\section{REFERENCES}

[1] V. Bach, T. Chen, J. Fröhlich, and I. M. Sigal, Smooth Feshbach map and operatortheoretic renormalization group methods, J. Funct. Anal. 203 (1), 44-92 (2003).

[2] T.Chen and N.Pavlović, On the Cauchy problem for focusing and defocusing GrossPitaevskii hierarchies, Discrete Contin. Dyn. Syst. 27 (2), 715-739 (2010).

[3] T.Chen and N.Pavlović, A new proof of existence of solutions for focusing and defocusing Groos-Pitaevskii hierarchies, arXiv:0906.3277.

[4] T.Chen and N.Pavlović, The quintic NLS as the mean field limit of a Boson gas with three-body interactions, J. Funct. Anal. 260 (4), 959 - 997 (2011).

[5] F.Dalfovo, S.Giorgini, L.P.Pitaevskii, and S.Stringari, Theory of Bose-Einstein condensation in trapped gases, Rev. Mod. Phys. 71, 463-512 (1999).

[6] A.Elgart, L.Erdös, B.Schlein, and H.T.Yau, Gross-Pitaevskii equation as the mean field limit of weakly coupled bosons, Arch. Rat. Mech. Anal. 179(2), 265-283 (2006).

[7] L.Erdös, B.Schlein, and H.T.Yau, Derivation of the Gross-Pitaevskii hierarchy for the dynamics of Bose-Einstein condensate, Commun. Pure Appl. Math. 59(12), 1659-1741 (2006).

[8] L.Erdös, B.Schlein, and H.T.Yau, Derivation of the cubic non-linear Schrödinger equation from quantum dynamics of many-body systems, Invent. Math. 167, 515614 (2007).

[9] L.Erdös, B.Schlein, and H.T.Yau, Derivation of the Gross-Pitaevskii equation for the dynamics of Bose-Einstein condensate, Ann. Math. to appear, arXiv:math$\mathrm{ph} / 0606017$.

[10] L.Erdös, B.Schlein, and H.T.Yau, Rigorous derivation of the Gross-Pitaevskii equation, Phys.Rev.Lett. 98, 040404 (2007).

[11] L.Erdös and H.T.Yau, Derivation of the nonlinear Schrödinger equation from a many body Coulomb system, Adv. Theor. Math. Phys. 5(6), 1169-1205 (2001).

[12] E.P.Gross, Structure of a quantized vortex in boson systems, Nuovo Cimento 20, 454-466 (1961).

[13] E.P.Gross, Hydrodynamics of a superfluid condensate, J.Math. Phys. 4, 195-207 (1963).

[14] S.Klainerman and M.Machedon, On the uniqueness of solutions to the GrossPitaevskii hierarchy, Commun.Math.Phys. 279, 169-185 (2008).

[15] E.H.Lieb and R.Seiringer, Proof of Bose-Einstein condensation for dilute trapped gases, Phys. Rev. Lett. 88, 170409 (2002).

[16] E.H.Lieb, R.Seiringer, and J.Yngvason, Bosons in a trap: A rigorous derivation of the Gross-Pitaevskii energy functional, Phys. Rev. A 61, 043602 (2000).

[17] E.H.Lieb, R.Seiringer, and J.Yngvason, A rigorous derivation of the Gross-Pitaevskii energy functional for a two-dimensional Bose gas, Commun. Math. Phys. 224, 17-31 (2001).

[18] L.P.Pitaevskii, Vortex lines in an imperfect Bose gas, Sov. Phys. JETP 13, 451-454 (1961). 
Wuhan Institute of Physics and Mathematics, Chinese Academy of Sciences, West District 30, Xiao-Hong-Shan, Wuhan 430071, China

School of Mathematics and Statistics, Central China Normal University, LuO-Yu RoAd 152, WuHAN 430079, China

E-mail address: chuangyeliu1130@126.com 